\title{
Evaporation rate of drop arrays within a digital microfluidic system
}

\author{
Laurent Davoust $^{\mathrm{a}, *}$, Johannes Theisen ${ }^{\mathrm{b}, \mathrm{a}}$

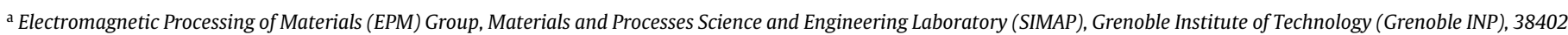 \\ Saint Martin d'Hères, France \\ ${ }^{\mathrm{b}}$ Laboratory of Geophysical and Industrial Fluid Flows (LEGI), University of Grenoble (UJF), 38041 Grenoble, France
}

\begin{abstract}
One essential advantage of digital microfluidic systems such as, for instance, drop-based lab-on-a-chips, is a massive parallelization of biochemical functions achieved by moving drops under surface acoustic waves or electrowetting on dielectrics (EWOD). This paper aims at characterizing the evaporation rate of a population of drops in a microsystem. Up to now, and despite its importance for end-users, the evaporation rate of one target drop selected among a population of drops has not been measured. This is essentially due to the difficulty of developing imaging in confined microfluidic drop arrays. In this paper, interferometry together with coplanar electrowetting are proposed as a new (non-imaging) evaporation rate measurement method fully compatible with digital microfluidic systems, and easy to be integrated into closed or open geometries. We investigate the impact on drop evaporation of different arrangements of drops and for different degrees of confinement. Use is made of dual-frequency electrowetting [1]: a classical actuation at a high-frequency $(15 \mathrm{kHz})$ allows to set the contact angle equal to $90^{\circ}$ while the low-frequency actuation $(100-500 \mathrm{~Hz})$ is responsible for the excitation of a perfectly spherical standing capillary wave along the drop surface. By considering natural frequencies of the oscillating drop and its capillary spectrum, it is possible to measure a spectral shift under evaporation. The evaporation law by Picknett and Bexon [2] is found valid even for a matrix of drops.
\end{abstract}

\section{Introduction}

In the past years, more and more applications of digital microfluidics using electrowetting on dielectrics (EWOD) [3,4] have emerged. Recent developments of electrowetting are concerned with lab-on-a-chips (LOC) [5], PCR [6,7], biological environmental monitoring [8] or cooling of electronic devices such as processor units and integrated circuits [9-11]. Depending on the application, evaporation is either not wanted, since there is the need to avoid mass loss (PCR), or deliberate since there is the need to gain benefit from phase change (cooling of PCBs). In all applications, it is therefore important to control evaporative mass transfer.

Digital microfluidic systems involve normally more than only one single drop, as their strong point is the possible parallelization of multiple functions in a drop array, which makes it difficult to properly evaluate evaporation via methods based on imaging or microscopy[12,13]. The impossibility of developing imaging is particularly important given that confinement is often deliberately intense for obvious reasons of miniaturization and portability. Top view imaging is only practicable in completely transparent devices, strictly limiting the choice of materials (e.g. ITO electrodes) and

\footnotetext{
* Corresponding author. Tel.: +33476825206.

E-mail address: laurent.davoust@simap.grenoble-inp.fr (L. Davoust).
}

thus LOC technology implementation. An alternative methodology for non-imaging control of evaporation is capacitive gauging of drop volume in a closed, or sandwich, electrowetting setup [14,15]. This technique was not yet developed for open, or coplanar, geometries, which should nevertheless be possible.

In this paper, we investigate the evaporative mass transfer of a drop in a microfluidics drop-array, as typically encountered in digital microfluidic systems involving heat transfers [16,17,11]. Different patterns are proposed to compare the mass transfer of a droplet in the center or at the edge of a drop pattern. Furthermore, different confinements are studied to examine the effects of a reduced drop array volume. Finally, the inter-drop distance is varied, providing information on proximity effects on evaporation.

The methodology presented in this paper is based both on EWOD and interferometry, to measure evaporation of a sessile drop, with a volume ranging between 0.5 and $10 \mu \mathrm{L}$ (see e.g. former work of the authors [1]). A dedicated electrowetting chip with coplanar electrodes [18] is used, which can be easily inserted in microsystems devoted to drop PCR with thermal cycling $[19,20]$ or to PCB/PCU cooling. Hydrophobic and dielectric layers on the electrodes acting in combination with electrowetting enable monitoring of an effective wetting angle for an aqueous drop at a constant value of $\theta=\pi / 2$ during the entire evaporation process. At the same time, the drop is brought to oscillate by perturbation of the actuation voltage between the buried electrodes. This technique is referred to as 


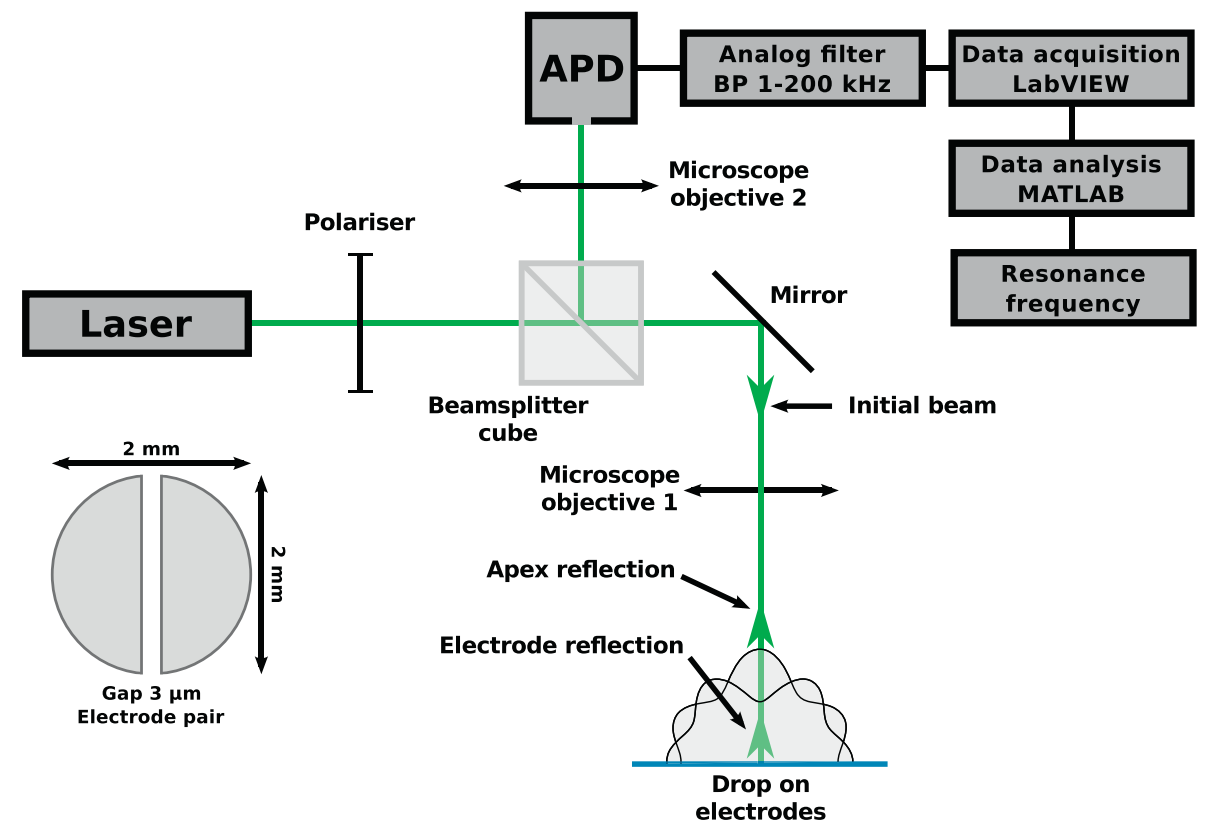

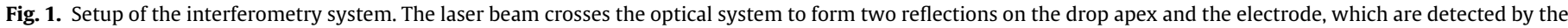
avalanche photo diode (APD). Insert: Electrode pair in use for electrowetting actuation.

dual-frequency electrowetting (DF-EW). The resonance spectrum of drop oscillation can be demonstrated to depend on the radius, which may be calculated progressively in the course of evaporation.

This methodology is fully compatible with integration requirements for microsystems such as digital lab-on-a-chips, as well as offering the advantage of permanently adjusting the contact angle (at $\theta=\pi / 2$ for instance, in this paper) using DF-EW.

\section{Dual-frequency coplanar electrowetting}

Electrowetting on dielectrics is a technology commonly used in new microfluidic applications such as liquid lenses [21] and EWOD-based displays [22]. A more recent application consists of a cooling methodology for active heat transfer management on processor units [10]. EWOD is also commonly used in digital labon-a-chips for moving, cutting/merging drops or mixing solubilized reagents among many operations $[23,24]$. Although a large part of bio-technological applications in lab-on-a-chips may be performed with silicone oil as a filler medium, some applications such as lyophilization or chemical noses still require an ambient gas phase [5]. In this case, the widely used sandwich configuration is not suitable, and a coplanar configuration is more adapted to different methodologies. For such systems, it is shown how EWODinduced drop shape oscillations can be provided and may easily be integrated in a closed or open microsystem.

It is observed that these oscillations form a convenient transduction mechanism for gauging evaporative mass transfer. As a matter of fact, an essential advantage of electrowetting is related to the lack of any ohmic current flowing within the drop: only electrostatic energy is stored in the underlying dielectric film. Thus, no Joule effect is expected to modify evaporation process at drop scale [25]. As recently demonstrated by Garcia-Sanchez et al. [26] for an AC electrowetting configuration, a second possible source of heat could be electrothermal effect due to a high frequency voltage ( $\sim 100 \mathrm{kHz}$ and beyond). But here, our highest frequency, $v$ (the one of the voltage $U_{1}$ ), never exceeds $15 \mathrm{kHz}$.

The coplanar electrowetting electrode geometry consists of two half-moons with a $2 \mathrm{~mm}$ base width facing each other with a $3 \mu \mathrm{m}$ gap (Fig. 1). The electrodes are covered by two coatings, an insulating layer $\left(\mathrm{Si}_{3} \mathrm{~N}_{4}\right.$, silicon nitride, thickness $d_{\mathrm{Si}_{3} \mathrm{~N}_{4}}=600 \mathrm{~nm}$, relative permittivity $\varepsilon_{\mathrm{Si}_{3} \mathrm{~N}_{4}}=6.3$ ) and a hydrophobic layer ( $\mathrm{SiOC}$, thickness $d_{\text {SiOC }}=1000 \mathrm{~nm}$, relative permittivity $\varepsilon_{\text {SiOC }}=2.75$ ). This technology has been demonstrated to exhibit excellent properties even in presence of high voltage, which is a prior feature as far as coplanar electrode designs are concerned [27]. The electrodes are covered by a very thin film of silicone oil (VWR Prolabo, viscosity $50 \mathrm{mPa}$ s) to avoid stick-slip motion, as well as an excessive value of contact angle hysteresis. The influence on electrowetting of the oil film after drainage is taken into account by estimating its thickness to $260 \mathrm{~nm}$ using standard wetting curve (see e.g. Fig. 2 in [1]). The amplitude of the high frequency EWOD voltage $U_{1}$ is chosen according to this wetting curve as to have a contact angle of $\theta=\pi / 2$.

The electrical potential applied to the electrode pair consists of two AC voltages, $U_{1}$ (frequency $v$ ) and $U_{2}$ (frequency $f$ ), provided by two frequency generators. These two signals are processed by an analog multiplier providing an output voltage, $U_{3}=\left(1+U_{2} / 10 \mathrm{~V}\right) U_{1}$. Voltage $U_{3}$ is further amplified by a high-voltage amplifier delivering dual-frequency electrowetting actuation, $U=100 U_{3}$. The electrode pair has one electrode connected to ground, while the other electrode is connected to voltage $U$.

The purpose of working with a dual-frequency actuation is that, when a high frequency, $v=15 \mathrm{kHz}$, is imposed on $U_{1}$, its amplitude may be used to tune the contact angle (for instance $\theta=\pi / 2$ ) in a range, which depends on composition of the underlying substrate, and to lower contact angle hysteresis [28]. Due to the minimization of electrostatic energy, voltage contribution $U_{1}$ means that the droplet straddles the underlying electrodes [18], making the droplet position far more stable than in standard geometries. Therein lies a second feature of prior importance, since interferometry measurements on a curved surface require a high degree of local accuracy to be feasible. Voltage modulation $U_{2}$ is characterized by a low frequency in the range of $f \sim 10-1000 \mathrm{~Hz}$. This voltage is used to radially oscillate the contact line of the drop, otherwise stabilized in position, and therefore to induce drop shape oscillations of a frequency $f_{\text {osc }}=2 f$, thereby controlling a purely standing capillary (spherical) wave on the drop surface. As demonstrated below, 
by changing the frequency $f$, it is easy to select capillary resonance ${ }^{1}$ with a given set of spherical standing wave modes along the drop surface.

In this work, the value of the electrowetting contact angle, $\theta$, is regulated to be equal to $\pi / 2$. This allows us to consider that the mean radius of the sessile droplet and the base radius are both equal, since, moreover, the gravity effect can be ignored in this case (Bond number small enough). As mentioned above, this regulation is made possible by adjusting the amplitude of $U_{1}$ according to the wetting curve displayed in [1] for $U_{2}=0$. Furthermore, the low-frequency electrowetting voltage, $U_{2}$, is chosen so as to generate a maximum amplitude of $20 \mu \mathrm{m}$, which permits first, to comply with supporting theory on linear drop oscillations and second, to avoid streaming current in ambient phase (air).

\section{Droplet interferometer}

A kind of Michelson interferometer is developed to detect surface motion at the apex of a sessile drop: a laser beam (wave length $\lambda=532 \mathrm{~nm}$ ) horizontally crosses a polarizer and a beam splitter, and is directed onto a mirror, where it is reflected vertically onto the drop apex underneath an inverted microscope objective (refer to Fig. 1 for a sketch of the optical setup). The liquid/gas interface results in two reflections: a first reflection of the laser beam at the drop apex, and a second reflection at the solid substrate, on which the drop rests. These two reflected beams take the same path back to the beam splitter, where they are directed through a second inverted microscope objective to collect maximum light intensity on the active surface of an avalanche photo diode, which is located at the focal point. The drop apex is well-suited for the interferometry measurement of the oscillation amplitude. The apex is the point where the amplitude of drop oscillations is largest and where oscillatory displacement must remain perfectly vertical. Thus, optimum sensitivity is expected at this point. Second, the apex is the only location along the drop surface, which remains parallel to the coplanar electrodes whilst translating vertically to them. Hence use is made of the drop apex as a semi-reflecting mirror, which has the advantage of directly working in the electrodes' reference system. This offers an elegant way of taking the interferometry measurement without need for an external mirror. With a precise optical alignment, i.e. a laser beam impacting at a right angle onto the electrodes, a reproducible and easy to process interferometry signal is obtained.

The delivered light intensity $I$ at the avalanche photo diode (APD) complies with the law[29]:

$I \propto I_{\mathrm{S}}+I_{\mathrm{a}}+2 \sqrt{I_{\mathrm{S}} I_{\mathrm{a}}} \cos \left(\frac{4 \pi}{L}\left[(\tilde{n}-1) \zeta(t)+\left(\tilde{n} R-\zeta_{0}(f)\right)\right]\right)$,

where $\tilde{n}$ is the refractive index of the liquid, $R$ is the instantaneous drop radius, ${ }^{2}$ and $I_{\mathrm{S}}$ and $I_{\mathrm{a}}$ are the light intensities received from the solid substrate and the drop apex, respectively. The oscillation amplitude of the vertically moving apex is $\zeta(t)=\zeta_{0}(f) \sin (4 \pi f t)$, where $f$ is the low frequency modulation of the EWOD actuation. The interferometry signal is frequency modulated by the velocity of the drop surface: Hence, the spectrum of the oscillation $\zeta_{0}(f)$ is related to the maximum frequency $f_{\mathrm{m}}$ of the interferometry signal:

$\zeta_{0}(f)=\frac{\lambda}{8 \pi(\tilde{n}+1)} \frac{f_{\mathrm{m}}}{f}$.

\footnotetext{
1 The spherical capillary wave excited from the oscillating contact line is propagating radially inwards up to drop apex where a wave interaction arises. In steady conditions, a resonance mechanism can be expected at the drop apex.

2 Here, the drop radius, $R$, is considered as instantaneous while being constant in the expression of light intensity since evaporation timescale is much larger than the timescale of the capillary wave, $1 / 2 f$.
}

The maximum frequency is evaluated via a routine, described in [30] and used in [31]. As far as signal processing of the interferometry signal is concerned, the APD signal is delivered to an analog filter (Krohn-Hite model 3362, BP $1 \mathrm{kHz} / 200 \mathrm{kHz}$, Butterworth type), prior to recording at a sampling frequency of $250 \mathrm{kHz}$. The data are subsequently subjected to post-processing to calculate an entire spectrum $\zeta_{0}(f)$ on the frequency range $f \sim f_{2} \pm 50 \mathrm{~Hz}$, for which the resonance frequency is evaluated. An example for the interferometry signal and a spectrum is given in [1]. Please refer to this reference for further details on the interferometry technique.

\section{Theoretical framework}

Consider a sessile, half-spherical water droplet with instantaneous radius, $R=R(t)$, whose initial value is $R_{0}$. It is worthy to recall here that the high frequency component of the EWOD voltage allows us to comply well with the experimental condition of a constant contact angle, $\theta=\pi / 2$, while evaporation is currently acting to downsize the droplet radially inwards. A well-known model of evaporation with a constant contact angle regime was presented by Picknett and Bexon [2], supported by more recent experimental developments by McHale et al. [32]. Since the effective contact angle is electrically adjusted to the value $\theta=\pi / 2$, the evolution of the radius can be written as follows:

$R(t)^{2}=R_{0}^{2}\left(1-\frac{t}{\tau_{\text {evap }}}\right)$

where $\tau_{\text {evap }}=R_{0}^{2} \rho /\left[4 D_{\text {vap }}\left(c_{0}-c_{\infty}\right)\right]$ is the characteristic evaporation timescale for diffusion-limited evaporation, $\rho$ is the density of water, $D_{\text {vap }}$ is the diffusion coefficient of the water vapor in the surrounding air, $c_{0}$ and $c_{\infty}$ are the mass concentrations in water vapor at the interface and far from it, respectively. In [1], surface stretching due to EWOD-induced drop oscillations is demonstrated to be negligible as far as its impact on mass transfer is concerned.

Droplet shape oscillations depend on surface tension and the averaged radius of the droplet. A sophisticated analysis by Kang et al. [33] delivers a theoretical model for the oscillations of a sessile drop in AC electrowetting. The electrical force in the wetting plane is modeled as a $\delta$-function at the triple contact line (TCL), $F_{\mathrm{TCL}}=c U^{2} / 8$, through which harmonic motions are established. This force depends on the applied voltage $U$ and on the resulting surface capacitance $c$ of the dielectric layers and the oil film. In this model, contact line friction and hysteresis are ignored. The amplitude of drop oscillations is consistently found to depend on excitation frequency $f$. The capillary resonance is demonstrated to occur for a given series of resonance frequencies, $f_{n}$, deduced from the classical dispersion relation:

$f_{n}=\frac{1}{4 \pi} \sqrt{\frac{\gamma \lambda_{n}(\theta)}{\rho R(t)^{3}}}$,

where $n=2 k$, and $k \in \mathbb{N}$, is the mode number of the oscillation of a sessile drop. In this paper, we introduce the factor $\lambda_{n}(\theta)$, which classically represents the eigenvalue $n(n-1)(n+2)$ for the oscillation of a free droplet, but which is modified in this case with the theory by Strani and Sabetta [34], devoted to the oscillation of a sessile drop with a contact angle $\theta$ (refer to [1] for further details). Consequently, from the dispersion relation (3), it is clearly demonstrated that the variation in radius can be converted into a resonance frequency shift, detected by means of interferometry.

\section{Proof of concept with one single drop}

Fig. 2(a) demonstrates the shifting of two resonance frequencies, namely $f_{2}$ and $f_{4}$, for a $5 \mu \mathrm{L}$ water drop under evaporation process (contact angle maintained at $90^{\circ}$ ). When drop radius diminishes, 


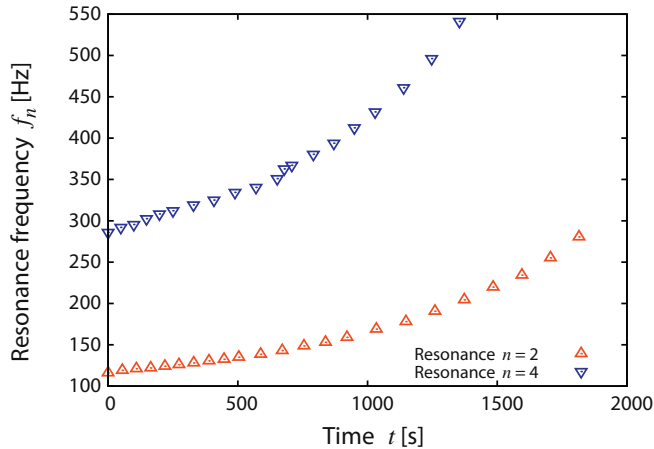

(a) Time dependence of the drop resonance frequencies $\mathrm{f}_{2}$ and $\mathrm{f}_{4}$.

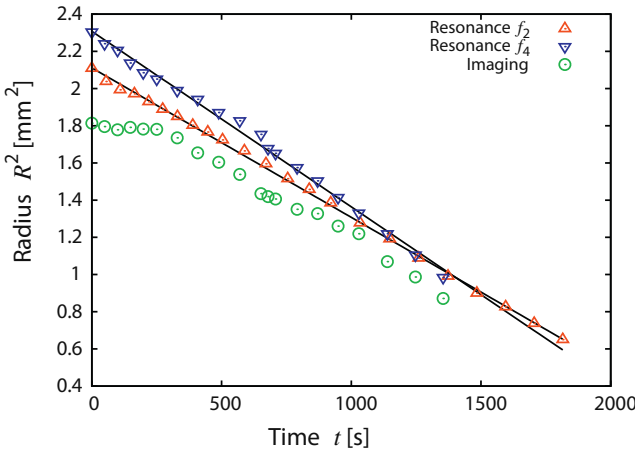

(b) The radius data is obtained via interferometry for mode $2(\Delta)$ and mode $4(\nabla)$, compared to imaging $(\mathrm{o})$. The measured evaporation times are $\tau_{\text {evap }, 2}=2627 \mathrm{~s}$ and $\tau_{\text {evap }, 4}=2445 \mathrm{~s}$ for mode 2 and 4 , respectively.

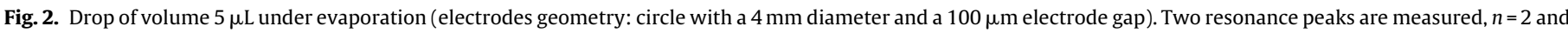

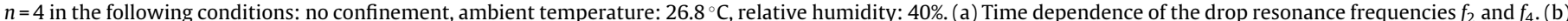

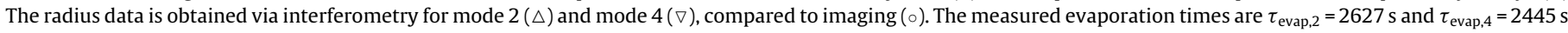
for modes 2 and 4, respectively.

the resonance frequencies shifts to higher and higher values, as expected from Eq. (3). The correction factors [34] for the second and fourth resonant modes can be calculated as $\lambda_{2}(\pi / 2)=8.3 .78$ and $\lambda_{4}(\pi / 2)=72 \cdot 3.32$, respectively. Fig. 2 (b) corresponds to the same measurement data, but the resonance frequencies are translated into a time-dependent radius by using Eq. (3). The slopes of the time-dependent resonance frequencies, especially the one corresponding to the fourth mode, exhibit a slight difference with the slope of the time-dependent drop radius as obtained from direct imaging of the drop. This discrepancy probably originates from the correction factors $\lambda_{n}(\theta)$, as already pointed out by Strani and
Sabetta [34]. A CCD camera (Basler) and a zoom lens (Optem Zoom 70XL) were used for imaging of the time-averaged drop. For lighting, use was made of a (blue) light emitting diode (LED).

\section{Drop arrays and impact of confinement}

\subsection{Liquid and geometries}

The drops with an initial volume of $1.5 \mu \mathrm{L}$ consist of ultra-pure water (resistivity $18.2 \mathrm{M} \Omega \mathrm{cm}$ ). The corresponding radius of a sessile drop is $R_{0}=0.895 \mathrm{~mm}$ for a contact angle of $\theta=\pi / 2$. The surface

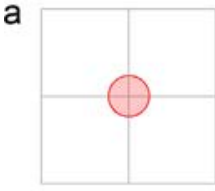

Config. 1

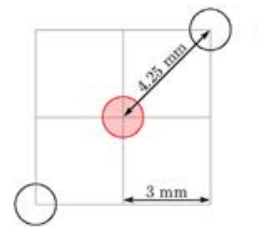

Config. 2

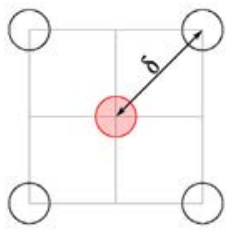

Config. 3

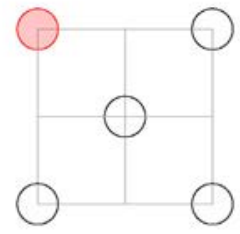

Config. 4

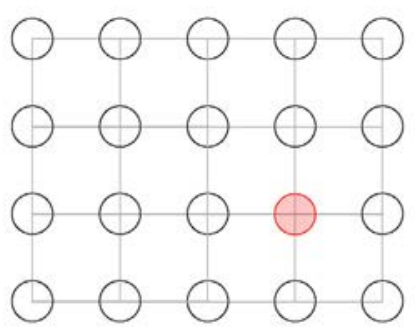

Config. 6 b

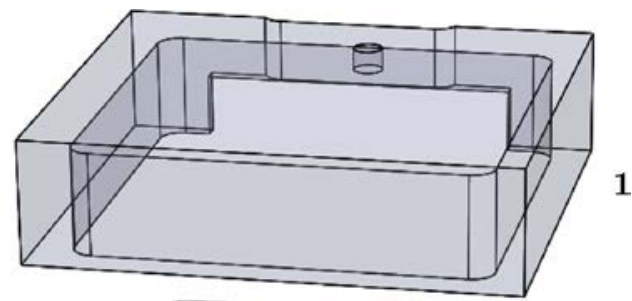

1
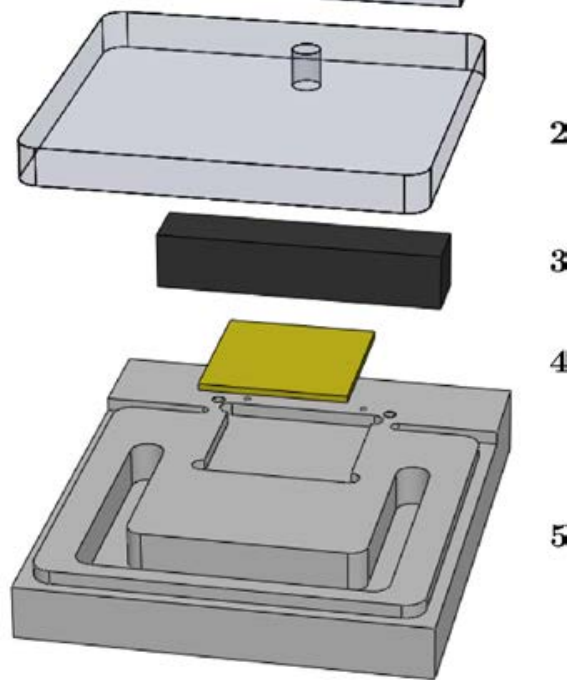

2

3

4

5

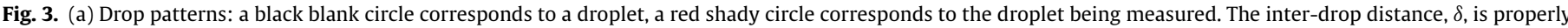

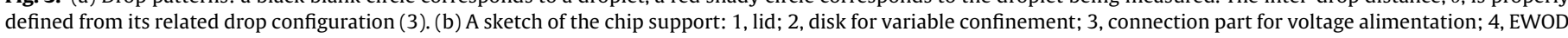
chip; 5 , chip support with $U$-shaped water bath. (For interpretation of the references to color in this figure legend, the reader is referred to the web version of the article.) 
Table 1

Confinement and inter-drop distances in units of $R_{0}$.

\begin{tabular}{lllllll}
\hline \multicolumn{2}{l}{ Confinement } & & & \multicolumn{3}{l}{ Inter-drop distance $\delta$} \\
\cline { 1 - 2 } \cline { 5 - 6 }$\#$ & In mm & In units of $R_{0}$ & & $\#$ & In mm & In units of $R_{0}$ \\
\hline b & 12 & 13.4 & & $1-6$ & 4.25 & 4.7 \\
c & 6.5 & 7.3 & & 7 & 8.5 & 9.5 \\
d & 3 & 3.4 & & 8 & 2 & 2.2 \\
\hline
\end{tabular}

tension is measured to be $57 \mathrm{mN} / \mathrm{m}$. Surface tension measurements have been performed with the Wilhelmy technique using a NIMA tensiometer. Due to traces of contamination, the surface tension is found to be lower than the expected theoretical value.

The chip with the electrodes is positioned on a Plexiglas support, which can be covered by a Plexiglas lid $(53 \mathrm{~mm} \times 56 \mathrm{~mm}$ bottom area, cf. sketch in Fig. 3(b)). The lid has an opening of $12 \mathrm{~mm}^{2}$ above the measured droplet to allow evaporation process to be completed within less than $1 \mathrm{~h}$. Into the lid can be inserted several slides of different thickness to adjust the vertical confinement.

Four confinements, $\kappa$, are tested: (a) no confinement (no lid, $\kappa \rightarrow \infty$ ), (b) a vertical confinement of $\kappa=12 \mathrm{~mm}$ (lid only), (c) a vertical confinement of $\kappa=6.5 \mathrm{~mm}$ and (d) a vertical confinement of $\kappa=3 \mathrm{~mm}$.

The drop arrays investigated are shown in Fig. 3(a)). A single droplet, a line of three droplets and a die-like five droplets arrangement are chosen. Drop patterns under consideration are namely: (1) a single drop, (2) a line of three drops, (3) an array of five drops with measurement at the center, (4) an array of five drops with measurement at the corner and (5) a single drop surrounded by a U-shaped channel filled with water (Fig. 3(b(5))). In addition, a drop pattern (6) is proposed: for the drop pattern (3), use is made of the U-shaped channel filled with water and moreover, sacrificial drops are added on the EWOD chip according to a $4 \times 5$ square grid with $3 \mathrm{~mm}$ side length to have an array of twenty drops on the chip (see e.g. Fig. 3(a) and (b)).

In order to estimate the impact of the inter-drop distance, $\delta$, on evaporation, drop pattern ( 3 ) is modified by doubling and reducing to the half, the distance between the drops $(\delta=8.5 \mathrm{~mm}$ and $\delta=2 \mathrm{~mm}$ for drop patterns ( 7 ) and (8), respectively, instead of $\delta=4.25 \mathrm{~mm}$ for drop pattern (3)). The inter-drop distance and confinement values are converted into units of $R_{0}$ in Table 1 .

The drops are deposited with a micro-pipette and the lid is closed immediately. The ambient relative humidity and temperature throughout the experiments are between $56 \%$ and $60 \%$ and $25.3^{\circ} \mathrm{C}$ and $26.8^{\circ} \mathrm{C}$, respectively.

\subsection{Results and discussion}

The different drop patterns, (1)-(8), are tested with different confinements, a-d, and the results are presented in Fig. 4 as well as in Table 2 in term of the evaporation time scale, $\tau_{\text {evap. The }}$ curves are non-dimensionalized by the initial radius, which has been extrapolated by a linear fit of $R(t)^{2}$. A distinct decline of mass

Table 2

Evaporation time scale $\tau_{\text {evap }}$ [s] for all confinements, a-d, and drop patterns, 1-8, considered in this paper.

\begin{tabular}{lcccc}
\hline Pattern/confinement & $\mathrm{a}(\kappa \rightarrow \infty)$ & $\mathrm{b}(\kappa=12 \mathrm{~mm})$ & $\mathrm{c}(\kappa=6.5 \mathrm{~mm})$ & $\mathrm{d}(\kappa=3 \mathrm{~mm})$ \\
\hline 1 & 1703 & 1653 & 2050 & 2161 \\
2 & 1966 & 2530 & 3410 & 3034 \\
3 & 2651 & 3610 & 3753 & 4192 \\
4 & 1959 & 2519 & 3761 & 3897 \\
5 & 1614 & 3998 & 3686 & 3215 \\
6 & - & - & - & 10,767 \\
7 & - & - & 2494 & - \\
8 & - & - & 4518 & - \\
\hline
\end{tabular}

loss due to evaporation is demonstrated when diminishing the vertical confinement from no confinement to $5 \mathrm{~mm}(\mathrm{a}-\mathrm{c})$. Beyond the confinement $c$, the slower evaporation rate is only significant for a small number of sacrificial drops.

\subsubsection{Drop patterns and confinements}

The comparison between drop pattern (3) with four sacrificial drops, and one single drop surrounded by a water bath (configuration 5), is only significant for low confinements (confinement a $(\kappa \rightarrow \infty)$ and confinement $\mathrm{b}(\kappa=12 \mathrm{~mm}))$. In both cases, the water bath efficiency is not clearly demonstrated. For stronger confinements, water bath and sacrificial droplets nearly exhibit the same efficiency in preventing evaporative mass loss $\left(\tau_{\text {evap }}^{\mathrm{d}-3} / \tau_{\text {evap }}^{\mathrm{d}-5}=1.3\right)$.

The 20 drops-based pattern with water bath (d-6), which exhibits the slowest evaporation kinetics, has a six-fold slower evaporation kinetics than a single drop without confinement, $\tau_{\text {evap }}^{\mathrm{d}-6} / \tau_{\text {evap }}^{\mathrm{a}-1}=6.3$. To consider the influence of the relative position on evaporation kinetics, one may compare drop pattern (3) to drop pattern (4). For weak confinements (a) and (b), there is a great difference in evaporation kinetics, whereas for strong confinements (c) and (d), the relative position of the drop does not strongly influence mass loss.

\subsubsection{Evaporation kinetics}

Concerning evaporation kinetics of the different drop patterns and confinements, one recognizes two different kinetics during the measurement. The evaporation time scale $\tau_{\text {evap }}$ is inversely proportional to the slope of the $R^{2}$-curve, assuming that the evaporation law (2) is valid. The first data points in Fig. 4 exhibit a slope slightly steeper than the one for the last (majority of) data points. When placing the lid onto the support, the drops follow first a non-confined evaporation regime at ambient temperature and humidity, as long as the humidity under the lid does not change significantly. When the thermodynamical equilibrium is reached, the evaporation regime changes: the water vapor exchange at the liquid surfaces (drop(s) + water bath as far as configurations 5 and 6 are concerned) is equal to the water vapor exchange between the inside and the outside of the lid, through the $12 \mathrm{~mm}^{2}$ hole. From our results, one can say that the initial evaporation regime is essentially characterized by drop pattern a-1. As the confinement and total initial water-air interface area is raised, the different evaporation kinetics will more and more tend to the kinetics of drop pattern d-6, which for the geometry of the confinement discussed in this paper, represents the slowest possible evaporation dynamics.

\subsubsection{Role of the inter-drop distance, $\delta$}

Concerning the study on the impact of the inter-drop distance, one may refer to the drop patterns c-3, c-7 and c-8, represented in Fig. 5. In comparison to the initial drop pattern c-3, drop pattern c-7 exhibits a time-ratio of $\tau_{\text {evap }}^{c-7} / \tau_{\text {evap }}^{c-3}=0.7$ (shorter evaporation time). Drop pattern c-8 exhibits a time-ratio of $\tau_{\text {evap }}^{c-8} / \tau_{\text {evap }}^{c-3}=$ 1.2 (longer evaporation time). The inter-droplet distance, $\delta$, thus impacts significantly on the evaporation time scale. This is though only true for a strong enough vertical confinement. In these cases, evaporation might be considered as being constrained to a horizontal 2D plane [35], which is a significant parameter for evaluating the effects of a modified inter-drop distance. ${ }^{3}$

\footnotetext{
${ }^{3}$ Regarding applications to EWOD microsytems, the minimum inter-drop distance is delivered by the geometrical characteristics of the electrode matrix (electrode size and electrode gaps) or by the actual distance between droplets moving along the electrode paths. Hence, these distances stand as decisive parameters for evaluating the effect of evaporation kinetics upon a drop array in a microsystem.
} 
(a)

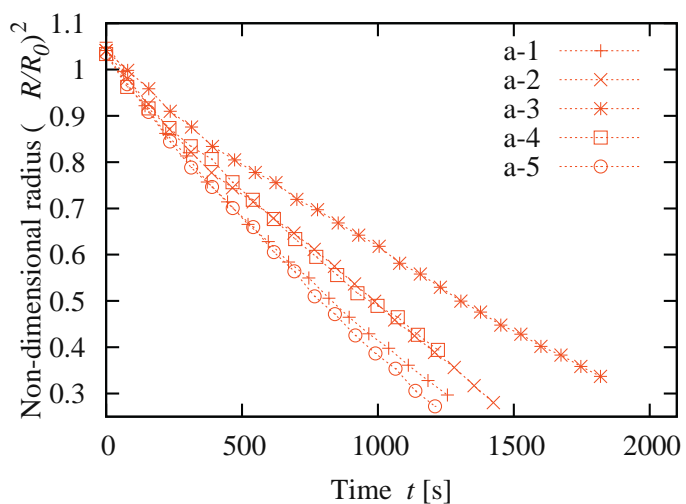

(c)

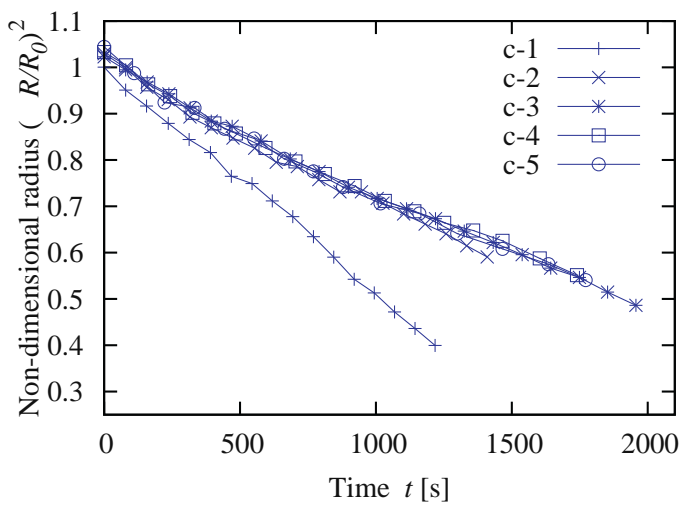

(b)

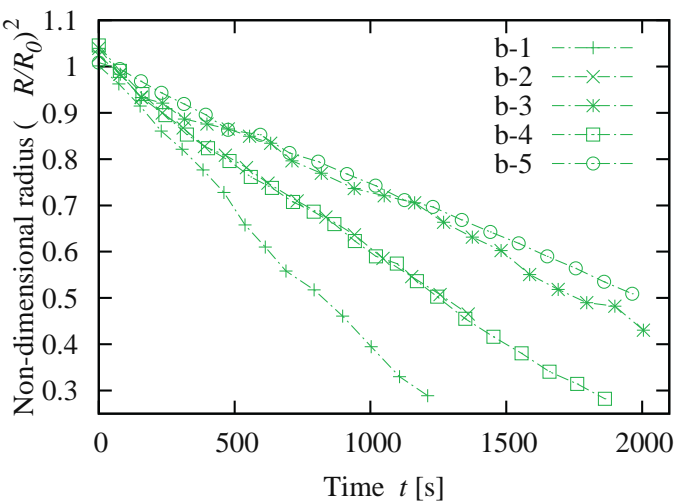

(d)

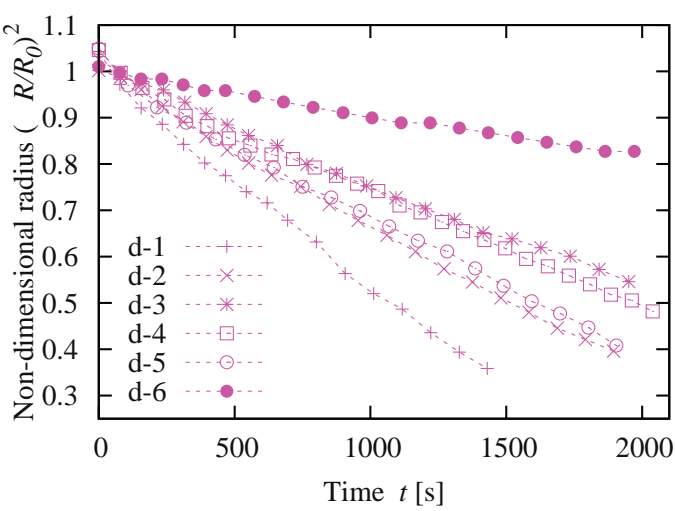

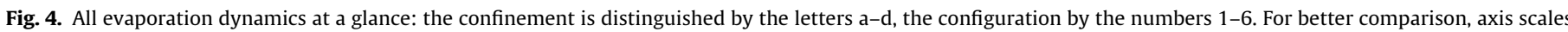
are the same on all graphs.

\subsubsection{Physical interpretation}

Fig. 6 shows the totality of the evaporation curves, nondimensionalized by $\tau_{\text {evap }}$ and the fitted initial radius. This graph shows that Eq. (2) is still valid for describing the evaporation of a population of drops in our confined system. The law (2) for evaporation is based on diffusion kinetics of the water vapor in the ambient gas phase. This leads to the evaporation time scale, $\tau_{\text {evap }}=R_{0}^{2} \rho /\left[4 D_{\text {vap }}\left(c_{0}-c_{\infty}\right)\right]$. When the inter-drop distance is altered, the governing factor for changing the evaporation time scale is the ambient vapor concentration $c_{\infty}$. This vapor mass concentration "far" from the drop surface is expected to increase when approaching the drops. It is necessarily calculated at mid-distance between two drops, as soon as the confinement is no longer the

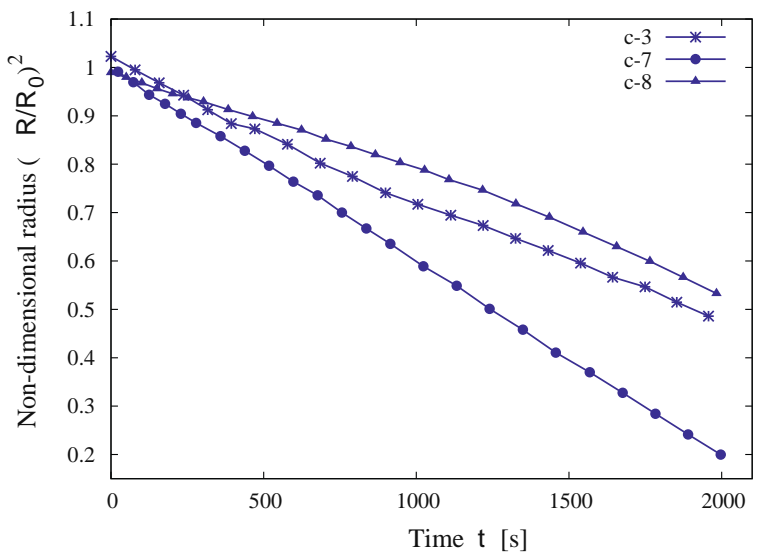

Fig. 5. Evaporation dynamics for drop patterns (7), (3) and (8) for a confinement of $\kappa=6.5 \mathrm{~mm}$ : the inter-drop distance is adjusted to $\delta=2 \mathrm{~mm}, 4.25 \mathrm{~mm}$ and $8.5 \mathrm{~mm}$, respectively.

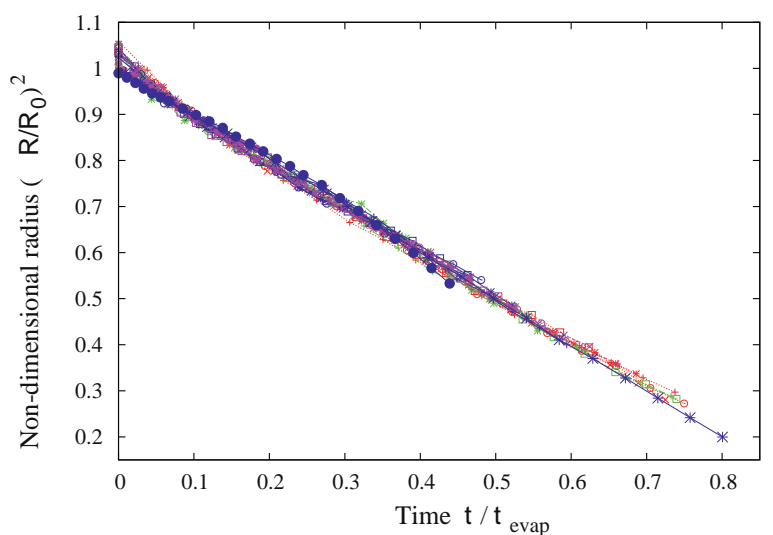

Fig. 6. Collapse of all experimental curves into one single curve according to nondimensionalized diffusive model (2).

limiting parameter. As a result, $\left(c_{0}-c_{\infty}\right)$ decreases and $\tau_{\text {evap }}$ increases. Conversely, $c_{\infty}$ is expected to lower when increasing the distance between neighboring drops. Other factors like $D_{\text {vap }}$ and $c_{0}$ should not be influenced so significantly.

\section{Conclusions}

Evaporation of drops in a microsystem can never be avoided since the volume can not be completely sealed with non permeable walls, for instance. This phenomenon is a key-issue for biological applications such as drop-based PCR with thermal cycling, for which the concentration of solubilized molecules and its dependence on evaporation must be anticipated.

Interferometry of drop shape oscillations sustained by dualfrequency electrowetting (DF-EW) has been shown to be a 
convenient measurement technique to capture the evaporation kinetics of a drop in a closed microsystem. In this paper, the timedependence of a half-spherical drop within a matrix of drops has been examined with respect to both the number of neighboring (sacrificial) drops and their proximity (inter-drop distance). The efficiency of limiting evaporation by making use of a water bath or sacrificial drops has been demonstrated to be dependent on the vertical confinement, $\kappa$. The even stronger influence of distance between drops, $\delta$, has been evaluated for three different values. The impact of $\delta$ upon evaporation kinetics is significant, especially when the strong enough confinement gives rise to a quasi-twodimensional evaporation process.

For slight confinements, the evaporation of a drop located at the edge of a five drop pattern is consistently found faster than the evaporation kinetics of a drop located at the center of the pattern. Conversely, for a strong enough confinement, the relative position of a target droplet (edge vs center) is no longer significant: all drops evaporate according to the same kinetics.

Finally, if the objective is to damp the evaporation process of one target drop, our experimental data suggest that the near presence of sacrificial drops must be preferred to the use of a liquid bath at a greater distance. As typical guidelines for end-users of digital microfluidic systems, it is worthy to note that for biological applications with millimeter-sized drops, the confinement needs to be smaller than $6 \mathrm{~mm}$, and any opening in the system leads inevitably to evaporation-driven mass loss, even for multiple drop patterns. For cooling applications involving phase change of millimeter-sized drops, the confinement should be at least $12 \mathrm{~mm}$ in order not to influence the evaporation kinetics and to have an efficient cooling effect.

The validity of the diffusive model developed originally by Picknett and Bexon for a drop with constant contact angle, is extended here to the case of one target drop, again with a constant angle, selected among a population of drops and whatever the confinement is. We know that surface wettability is a determining parameter for the evaporation of a sessile drop [36]. In this paper, we have studied only one contact angle $(\theta=\pi / 2)$ but it is easy to set another value of the contact angle by changing the high frequency voltage component of DF-EW. This ability of DF-EW delivers interesting outlooks for any other evaporation processes. Typically, the two classical evaporation regimes [2] can be applied: constant contact angle mode or constant contact radius mode.

As far as integration purposes are concerned, a practical advantage of jointly using interferometry and DF-EW is the possible measurement of evaporation in a confined environment with a drop distribution which might hinder proper imaging. This situation is usually encountered in lab-on-a-chips, as parallelization of tasks is one of the main goals.

\section{Acknowledgements}

The authors are grateful to Dr. Yves Fouillet (CEA-Grenoble/LETI) and Dr. Rachid Malk (CEA-Grenoble/LETI and Grenoble-INP/LEGI Laboratory) for fruitful discussions on electrowetting, electronic packaging and chips loan.

\section{References}

[1] J. Theisen, L. Davoust, Dual-frequency electrowetting: application to drop evaporation gauging within a digital microsystem, Langmuir 28 (1) (2012) 1041-1048.

[2] R.G. Picknett, R. Bexon, The evaporation of sessile or pendant drops in still air, Journal of Colloid and Interface Science 61 (2) (1977) 336-350.

[3] B. Berge, C. Quilliet, Electrowetting: a recent outbreak, Current Opinion in Colloid \& Interface Science 6 (2001) 34-39.

[4] F. Mugele, J.-C. Baret, Electrowetting: from basics to applications, Journal of Physics: Condensed Matter 17 (2005) R705-R774.
[5] M.G. Pollack, V.K. Pamula, V. Srinivasan, A.E. Eckhardt, Applications of electrowetting-based digital microfluidics in clinical diagnostics, Expert Review of Molecular Diagnostics 11 (4) (2011) 393-407.

[6] M.G. Pollack, P.Y. Paik, A.D. Shenderog V.K. Pamula, F.S. Dietrich, R.B. Fair, Investigation of electrowetting-based microfluidics for real-time PCR applications, in: Proceedings of the 7th Int. $\mu$ TAS Conf., vol. 1, 2003, pp. 619-622.

[7] T. Zhang, K. Chakrabarty, R.B. Fair, Behavioral modeling and performance evaluation of microelectrofluidics-based PCR systems using systemC, IEEE Transactions on Computer-Aided Design of Integrated Circuits and Systems 23 (2004) 843-858.

[8] C. Delattre, C. Allier, Y. Fouillet, D. Jary, F. Bottausci, D. Bouvier, G. Delapierre, M. Quinaud, A. Rival, L. Davoust, C. Peponnet, Macro to microfluidics system for biological environmental monitoring, Biosensors and Bioelectronics 36 (1) (2012) 230-235

[9] K. Mohseni, E.S. Baird, Digitized heat transfer using electrowetting on dielectrics, Nanoscale and Microscale Thermophysical Engineering (2007) 99-108.

[10] J.-T. Cheng, C.-L. Chen, Active thermal management of on-chip hot spots using EWOD-driven droplet microfluidics, Experiments in Fluids 49 (2010) 1349-1357.

[11] N. Kumari, S.V. Garimella, Characterization of the heat transfer accompanying electrowetting or gravity-induced droplet motion, International Journal of Heat and Mass Transfer 54 (2011) 4037-4050.

[12] N. Zhang, D.F. Chao, A new approach to measure contact angle and evaporation rate with flow visualization in a sessile drop, NASA/TM-1999-209636, 1999.

[13] O.D. Gazzola, B. Iafelice, E. Jung, E. Franchi, R. Guerrieri, An integrated electronic meniscus sensor for measurement of evaporative flow, in: Solid-State Sensors, Actuators and Microsystems Conference, 2007. TRANSDUCERS 2007. International, June 2007, pp. 1805-1808.

[14] J. Gong, C.J. Kim, All-electronic droplet generation on-chip with real-time feedback control for EWOD digital microfluidics, Lab on a Chip 8 (2008) 898-906.

[15] S. Sadeghi, H. Ding, G.J. Shah, S. Chen, P.Y. Keng, C.J. Kim, R.M. van Dam, On chip droplet characterization: a practical, high-sensitivity measurement of droplet impedance in digital microfluidics, Analytical Chemistry 84 (2012) 1915-1923.

[16] V.K. Pamula, K. Chakrabarty, Cooling of integrated circuits using droplet-based microfluidics, in: Proceedings of the ACM Great Lakes Symposium on VLSI, 2003, pp. 84-87.

[17] P. Paik, V.K. Pamula, K. Chakrabarty, Adaptive hot-spot cooling of integrated circuits using digital microfluidics, in: Proceedings of IMECE, FL, USA, IMECE2005-81081, 2005

[18] P. Sen, C.-J. Kim, Capillary spreading dynamics of electrowetted sessile droplets in air, Langmuir 25 (8) (2009) 4302-4305.

[19] F. Wanget, A. Burns, Performance of nanoliter-sized droplet-based microfluidic PCR, Biomedical Microdevices 11 (2009) 1071-1080.

[20] A.C. Hatch, J.S. Fisher, A.R. Tovar, A.T. Hsieh, R. Lin, S.L. Pentoney, D.L. Yang, A.P. Lee, 1-Million droplet array with wide-field fluorescence imaging for digital PCR, Lab on a Chip 11 (2011) 3838-3845.

[21] B. Berge, J. Peseux, Variable focal lens controlled by an external voltage: an application of electrowetting, European Physical Journal E 3 (2000) 159-163.

[22] R.A. Hayes, B.J. Feenstra, Video-speed electronic paper based on electrowetting, Nature 425 (2003) 383-385.

[23] R.B. Fair, Digital microfluidics: is a true lab-on-a-chip possible? Microfluidics and Nanofluidics 3 (2007) 245-281.

[24] R.B. Fair, M.G. Pollack, A.D. Shenderov, Electrowetting-based actuation of droplets for integrated microfluidics, Lab on a Chip 2 (2002) 96-101.

[25] T.B. Jones, K.-L. Wang, D.-J. Yao, Frequency-dependent electromechanics of aqueous liquids: electrowetting and dielectrophoresis, Langmuir 20 (7) (2004) 2813-2818.

[26] P. Garcia-Sanchez, A. Ramos, F. Mugele, Electrothermally driven flows in ac electrowetting, Physical Review E 81 (2010) 015303.

[27] R. Malk, Y. Fouillet, L. Davoust, Rotating flow within a droplet actuated with AC EWOD, Sensors and Actuators B 154 (2011) 191-198.

[28] F. Li, F. Mugele, How to make sticky surfaces slippery: contact angle hysteresis in electrowetting with alternating voltage, Applied Physics Letters 92 (2008) 244108.

[29] L. Davoust, J.L. Achard, A. Cartellier, Detection of waves at an interface by way of an optical fibre, Progress in Colloid and Polymer Science 115 (2000) 249-254.

[30] C. Picard, L. Davoust, A "fluid cantilever" to detect amphiphilic biomolecules, Colloids and Surfaces A: Physicochemical and Engineering Aspects 343 (2009) 12-19.

[31] C. Picard, L. Davoust, Resonance frequencies of meniscus waves as a physical mechanism for a DNA biosensor, Langmuir 23 (2007) 1394-1402.

[32] G. McHale, S.M. Rowan, M.I. Newton, M.K. Banerjee, Evaporation and the wetting of a low-energy solid surface, Journal of Physical Chemistry B 102 (1998) 1964-1967.

[33] K.H. Kang, J.M. Oh, S.H. Ko, Shape oscillation of a drop in AC electrowetting, Langmuir 23 (15) (2008) 8379-8386.

[34] M. Strani, F. Sabetta, Free vibrations of a drop in partial contact with a solid support, Journal of Fluid Mechanics 141 (1984) 233-247.

[35] D.J. Harris, J.C. Conrad, J.A. Lewis, Evaporative lithographic patterning of binary colloidal films, Philosophical Transactions of the Royal Society of London Series A: Mathematical Physical and Engineering Sciences 367 (2009) 51575165.

[36] H. Song, Y. Lee, S. Jin, H.-Y. Kim, J.Y. Yoo, Prediction of sessile drop evaporation considering surface wettability, Microelectronic Engineering 88 (11) (2011) 3249-3255. 


\section{Biographies}

Laurent Davoust received his Engineer diploma in mechanical engineering and his PhD in buoyancy-driven MHD flows from the Institut National Polytechnique de Grenoble (INPG) in 1993 and 1996, respectively. In 1997, he joined CNRS as a permanent researcher (LEGI Laboratory). In 2010, he joined Grenoble Institute of Technology (Grenoble-INP) as a Professor in fluid mechanics, transport phenomena.
His current research topics include microfluidics, fluid interfaces and particles with some developments in digital microfluidics (lab-on-a-chips), in nanoimprint of polymer thin films and in electro-magneto-hydrodynamics.

Johannes Theisen received his Engineer diploma in applied physics at Grenoble-INP in 2009. He also received his diplomarbeit at KIT in 2010. From 2009 to end of 2012 he was working toward a PhD degree at the LEGI laboratory in Grenoble. He recently received his PhD on microfluidics in February 2013 at the University of Grenoble. 